\title{
ARTICLE
}

\section{A clinical update on paediatric lupus}

\author{
G W Spittal, ${ }^{1}$ MB ChB (Glasgow, UK), MRCPCH (UK), FCPaed (SA), MMed Paed, Dip HIV Man; L B Lewandowski, ${ }^{2}$ MD, MS; \\ C Scott, ${ }^{1}$ MB ChB, FCPaed (SA), Grad Cert Paed Rheum (UWA)
}

\author{
${ }^{1}$ Paediatric Rheumatology, Red Cross War Memorial Children's Hospital, University of Cape Town, South Africa \\ ${ }_{2}^{2}$ Pediatric Rheumatology, Duke University Medical Center, Durham, NC, USA
}

Corresponding author: GW Spittal (gspittal@hotmail.com)

Systemic lupus erythematosus in children is a life-threatening chronic disease that is being increasingly recognised. More black African children are being diagnosed and the proportion of males affected is much higher than in adult-onset lupus. The presenting manifestations of childhood-onset lupus are variable and many systems are involved. Children with lupus often present late with severe disease, and in South African (SA) children severe lupus nephritis occurs commonly at presentation. The investigations for lupus should be performed in a three-step process - initial essential investigations, antibody and serological tests, and supplementary investigations.

The most important factor in the management is to involve a multidisciplinary team as soon as possible. All cases of lupus in SA should be discussed with a paediatric specialist so that a tailored management plan can be made, depending on the presenting features and course of the disease.

S Afr Med J 2015;105(12):1075. DOI:10.7196/SAMJ.v105i12.10341

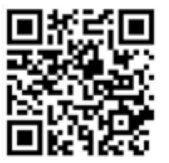

Systemic lupus erythematosus (SLE) is a multisystem, inflammatory, autoimmune disease characterised by the formation of antinuclear antibodies. ${ }^{[1]}$ It is one of the most common autoimmune diseases, with an estimated incidence of $2.0-7.6$ per $100000 .{ }^{[2,3]}$ Because SLE can present in a multitude of ways that mimic other diseases, early diagnosis can be difficult.

\section{Methods}

- A literature review from the first author's MMed dissertation was used as the main reference source.

- PubMed and Google scholar searches were conducted to identify more recent publications. Abstracts from 2014 and 2015 were reviewed and relevant full-text articles were read and crossreferenced.

- Keywords were the following: Systemic Lupus Erythematosus; SLE; Children; Update; Management; and Autoantibodies.

- Personal reference lists of the 3 authors were used in this update.

- Most of the data were either level B or level C.

\section{Who presents with the disease?}

Although more commonly present in adults, childhood-onset SLE (cSLE) is becoming increasingly recognised, with approximately $15-20 \%$ of SLE occurring before the age of 19 years. ${ }^{[1,4]}$ The presentation of SLE differs, not only with regard to age, but also depending on the ethnic background and gender of the patient. ${ }^{[2,5]}$ The disease has been reported to be more common in Europe, Asia and the USA than in Africa; yet, patients of African ancestry living in these regions have the highest rates of SLE. ${ }^{[6]}$ There is increasing recognition that SLE is not as rare in Africa as previously thought. The discrepancy may be related to many factors, including underdiagnosis due to poor access to healthcare and under-recognition of the disease. ${ }^{[7]}$

More females than males are affected with SLE at all ages, but the male to female ratio varies. The highest incidence is among women of childbearing age, with the female to male ratio from puberty to menopause being about 9:1. However, in prepubertal and postmenopausal patients, this ratio is about $4: 1 .^{[2,4]}$

\section{What causes lupus?}

The aetiology of SLE is not fully understood. Genetic, environmental, immunological and infective factors are all thought to play a role..$^{[1]} \mathrm{It}$ is hypothesised that a trigger acts on genetically susceptible individuals, and complex immune dysregulation of multiple components of the immune system as well as disordered apoptosis result in the production of autoantibodies that are particularly active against proteins in the cell nucleus. Recent evidence is emerging to illustrate the intertwined roles of the adaptive and innate immune system in this disease, and type 1 interferon has been shown to play a prominent role in the development of SLE. ${ }^{[8]}$

\section{What diagnostic criteria exist?}

SLE is diagnosed clinically and in the laboratory. It is based on the American College of Rheumatology (ACR) Classification Criteria for SLE, which were revised in $1997 .{ }^{[9]}$ Four of the 11 criteria are required to make the diagnosis. These criteria were established mainly for use in scientific research; therefore many children, especially those with lupus nephritis (LN) or antiphospholipid syndrome, might have SLE without fulfilling four of the criteria.

The Systemic Lupus International Collaborating Clinics (SLICC) Classification Criteria for SLE have recently been proposed and validated in adults with lupus, and have been shown to have greater sensitivity but lower specificity compared with the ACR criteria. It requires four criteria with at least one clinical and one immunological criterion to be present to make the diagnosis. ${ }^{[10]}$

\section{How do children with lupus present?}

Globally, the presenting features differ, which is one of the reasons that SLE can be difficult to diagnose. It remains problematic to establish whether a specific symptom or sign will occur in any individual or any group of patients, although cSLE is known to be more severe at diagnosis when compared with adult-onset SLE.

In 2005, a cohort of 36 patients from Gauteng, including 14 black African children, was reported on, concluding that SLE is being increasingly recognised in black SA children. ${ }^{[11]}$

A recent study from Cape Town reports on 68 patients, with a median age of 12.2 years and an approximate female to male ratio of 5:1. The 
study included a larger proportion of black and coloured patients than previous studies. This cohort of patients presented with severe disease at diagnosis, with most of them having LN. Preliminary findings show these children presenting with high disease activity and progression to end-organ damage at higher rates than in developed nations. ${ }^{[12]}$

Table 1 summarises the common clinical features of cSLE. Almost all children present with constitutional symptoms, e.g. fever, lymphadenopathy and weight loss, mimicking tuberculosis (TB) and HIV in our setting. ${ }^{[13]}$ Worldwide, common presenting features in cSLE are arthritis, malar rash and renal involvement. . $^{[4,13,14]}$

In SA, cutaneous (77\%) and constitutional $(55.5 \%)$ features as well as renal involvement (44-50\%) have been shown to be common presenting features. Other presenting features in SA children included serositis and Raynaud's phenomenon. ${ }^{[11,12]}$

SLE presenting in childhood seems to have more frequent renal and neurological organ involvement, which heavily influences prognosis and therapy. It is essential to screen all children with SLE for these major manifestations. ${ }^{[4,11]}$

\section{Which investigations should be performed?}

When investigating children with suspected SLE, the ACR criteria may be used to guide the initial investigations. Table 2 gives an outline of the investigations in a stepwise pattern.

Antinuclear antibody (ANA) testing and anti-extractable nuclear antigen form the mainstay of serological testing for SLE. Autoantibodies directed to nuclear antigens consist of various types of antibodies characterised by different antigen specificities. These nuclear antigens include single- and double-stranded DNA (ds DNA), histone proteins (histone DNA complex), nucleosome, centromere proteins and extractable nuclear antigens (including antiSmith, Ro, La, ribonucleoprotein). Understanding the specificities of the antibodies is useful in clinical practice to further classify patients with lupus and help predict their disease course. ${ }^{[15,16]}$

\section{Management}

The management of cSLE is difficult and referral to specialists in this field is essential. While there is no single cure for the disease, the aim of therapy at all stages of cSLE is to maximise the therapeutic effect while minimising disease activity and adverse effects. It is important to base treatment decisions on the systems/organs involved and the severity of the disease. SA is still

Table 1. Common clinical features of cSLE

\begin{tabular}{lll}
\hline & \multicolumn{2}{c}{ Estimated presence of involvement, \% } \\
\cline { 2 - 3 } Clinical feature & Paediatric SLE literature ${ }^{[4,13,14]}$ & South Africa ${ }^{[1,12]}$ \\
\hline Any constitutional feature & 100 & 55.5 \\
Fever & 39 & \\
Lymphadenopathy & $11-20$ & 33.3 \\
Arthritis & $61-64$ & 38.8 \\
Nephritis & $28-62$ & 44.4 \\
Mucocutaneous & & 77 \\
Malar rash & $55-62$ & 47 \\
Neuropsychiatric & $15-20$ & 19
\end{tabular}

trying to recover from the apartheid system and its inequalities in healthcare provision. ${ }^{[17]}$ Recommendations can be made to offer our children the most appropriate management.

The approach to management can be thought of as a 6-step process: using the multidisciplinary team (MDT), initial induction treatment, maintenance therapy, adjuvant therapy, managing disease flares, and disease monitoring. ${ }^{[8,18,19]}$

\section{Multidisciplinary team}

All children with SLE should be managed in a specialist paediatric centre, preferably with access to paediatric rheumatologists, paediatric nephrologists and appropriate support staff. A comprehensive MDT is essential. Patients should be assessed individually and their specific needs addressed on a patient-to-patient basis. SLE is a life-long condition with a natural course of chronicity, with disease flares and accumulation of organ damage. Anticipating potential problems and planning for these in advance can help to reduce the impact of the disease on the patient and the child's family.

Adolescence is a dangerous time for all patients with chronic medical conditions, and lupus is no exception. There are many potential pitfalls at this time, including non-compliance, recreational drug use, psychosocial issues and pregnancy, which should be addressed. The transitional process through adolescence into adulthood should be carefully planned, with the involvement of the patient, their family and the MDT.

\section{Initial induction treatment}

Induction treatment should only be given after discussion with paediatric specialists, preferably those with knowledge of cSLE.

This usually occurs in the first 6 - 12 months after diagnosis and is based on the use of high-dose immunosuppressive therapy to achieve disease stabilisation. Currently this is done initially with intravenous methylprednisolone $(30 \mathrm{mg} / \mathrm{kg}$ per dose for 3 days), together with immunosuppressive therapy and disease-modifying drugs. Weaning of the corticosteroid dose must be gradual and supervised carefully by an experienced team.

Although the use of corticosteroids remains the first line of treatment, it is essential that every effort be made to minimise the dosage because of the extensive adverse effect profile. Steroid-free induction regimens are being developed and show some early promise as an appropriate treatment option, while reducing sideeffects. ${ }^{[8,18,19]}$

Long-term efficacy has been demonstrated for intravenous cyclophosphamide-based regimens $\left(500-750 \mathrm{mg} / \mathrm{m}^{2}\right.$ per month for 6 months). It can be particularly useful in patients with neurological, renal or systemic vasculitis and is more readily available in SA compared with some of the other immunosuppressive medications. ${ }^{[8,19]}$

Mycophenolate mofetil (MMF) is an alternative treatment option and recent trials have demonstrated similar efficacy to cyclophosphamide, especially in patients with moderate to severe forms of LN, with a more favourable toxicity profile. ${ }^{[8,19]}$

Azathioprine and methotrexate are other options available in SA. They are both used with corticosteroids in patients with mild to moderate disease. Azathioprine is useful in maintenance therapy for patients with LN and can be used to delay the presentation of neuropsychiatric manifestations of SLE. Methotrexate has been shown to be an effective agent in controlling cutaneous and articular manifestations of the condition. ${ }^{[19]}$

Newer biologics are currently being used for the treatment of cSLE, but are not readily available in SA. ${ }^{[20]}$ Belimumab is the only biologic drug registered by the US Food and Drug Administration for use in SLE. It is a monoclonal antibody to the soluble human B lymphocyte stimulator protein and is the 
Table 2. Outline of investigations in stepwise pattern

\begin{tabular}{|c|c|}
\hline Investigation & Explanation \\
\hline \multicolumn{2}{|l|}{ Initial essential investigations } \\
\hline Full blood count & For anaemia, leucopenia and thrombocytopenia \\
\hline Electrolytes, urea and creatinine & Assess the renal function \\
\hline $\begin{array}{l}\text { Urine dipstick, microscopy, protein: creatinine } \\
\text { ratio and urinary red blood cell casts }\end{array}$ & $\begin{array}{l}\text { Check for both haematuria and proteinuria. } \mathrm{LN} \text { is commonly present at diagnosis in childre } \\
\text { especially in African children, and impacts on the initial management and long-term morbic } \\
\text { and mortality }\end{array}$ \\
\hline $\begin{array}{l}\text { Complement levels - C3, C4 and total } \\
\text { complement levels }\end{array}$ & Often reduced in SLE \\
\hline ESR or CRP & $\begin{array}{l}\text { Indicates current inflammation } \\
\text { The level of ESR elevation may show a discrepancy relative to a normal CRP level in flares; } \\
\text { if both markers are elevated, suspect the presence of an infective process }\end{array}$ \\
\hline
\end{tabular}

Autoantibody and serological tests - immunological tests looking for autoantibodies should be performed

ANA

Anti-ds DNA antibody

Anti-Smith antibody

Antiphospholipid antibodies: anticardiolipin, $\beta_{2}$-glycoprotein-1 and lupus anticoagulant Antinucleosome antibodies

Anti-Ro and anti-La antibody

Syphilis test
These are the common autoantibodies found in patients with SLE - used as a screening test. By definition, all patients should be ANA positive at diagnosis. However, there are a small proportion of patients who are negative at diagnosis and become positive later in the disease. Not specific for SLE, as up to $30 \%$ of the population are low-titre positive

High specificity but sensitivity only $70 \%$. Level varies depending on disease activity, so can be used to monitor the clinical course. Shown to be increased in patients with LN and in neuropsychiatric lupus

Specific but not sensitive marker of SLE. Reactivity is not described in other diseases Associated with the anti-phospholipid syndrome, cerebral vascular disease and neuropsychiatric lupus

The first serological marker of SLE described and considered a major autoantigen in SLE positive in about $85 \%$. An excellent marker for SLE, playing an important role in pathogenesis. A good predictor of flares

Particularly relevant in female adolescents who are considering having children. Known to play a role in the cardiac manifestations of neonatal lupus and photosensitivity

A false-positive serological test for syphilis is also in the ACR diagnostic criteria for SLE

\section{Supplementary investigations, depending on history, examination and presenting clinical features}

Liver function tests

Haemolysis work-up - includes a reticulocyte count, lactate dehydrogenase, haptoglobin, blood smear and a direct Coombs test

Renal biopsy

Chest radiograph

Electrocardiogram

Echocardiography

Tuberculin skin test

Ophthalmological examination

Lumbar puncture

Brain magnetic resonance imaging/magnetic resonance angiography

Skin biopsy

CK
May be mildly elevated in acute SLE

Perform if the haemoglobin level is low and/or haemolysis is suspected

If renal involvement is present, a renal biopsy may be necessary. These should be classified according to the International Society of Nephrology and the Renal Pathology Society staging score

For pericardial or pleural effusion, evidence of pericarditis, interstitial lung disease, pneumonitis and pulmonary embolism. In SA it is useful to look specifically for any radiological signs of tuberculosis For evidence of pericarditis or pericardial effusion

For pericardial effusion or pulmonary hypertension

Can be used mainly in younger patients to screen for tuberculosis

For evidence of optic neuritis

To exclude infection with fever or neurological symptoms or signs. Nonspecific elevations in cell count and protein level and a decrease in glucose level may be found in the cerebrospinal fluid of patients with CNS lupus

To evaluate for CNS lupus white matter changes, vasculitis or stroke

Can be helpful in diagnosing SLE in patients with unusual skin rashes. Many different rashes may indicate SLE and review by a dermatologist is important

May be elevated in myositis

$\mathrm{ESR}=$ erythrocyte sedimentation rate; $\mathrm{CRP}=\mathrm{C}$-reactive protein; $\mathrm{ANA}=$ antinuclear antibody $\mathrm{CNS}=$ central nervous system; $\mathrm{CK}=$ creatinine kinase $\mathrm{LN}=$ lupus nephritis 
only biologic agent to demonstrate efficacy in adult SLE randomised controlled trials. Studies involving children are currently underway. Rituximab is currently being used off-label for select patients in the SA public health sector. It is commonly used for patients with SLE, despite the optimal dose of administration and indications of treatment being uncertain. ${ }^{[8,20]}$

Although not routine, plasma exchange and intravenous immunoglobulin therapy are sometimes used by specialist physicians with experience of cSLE for specific indications. The main indications are in rapidly progressive life-threatening disease, neuropsychiatric lupus refractory to conventional treatment and idiopathic thrombocytopenic purpura. ${ }^{[19]}$

\section{Maintenance therapy}

Ongoing maintenance therapy is usually with weaning doses of oral corticosteroids in conjunction with disease-modifying agents as discussed above. Cyclophosphamide, MMF, azathioprine and rituximab are used, depending on disease severity and systems involved. Maintenance therapy has to be continued for several years to achieve ongoing disease remission and prevent steroid toxicity. ${ }^{[8,18,19]}$

\section{Adjuvant therapy}

An often overlooked key factor in the care of children with SLE is the importance of adjuvant therapy (Table 3).

\section{Managing disease flares}

Disease flares are a common occurrence in cSLE. They should be screened for and managed aggressively according to the severity of presentation and organ system involved. It is important to address compliance, changes in the social situation, as well as patient and family psychosocial well-being. Drug doses should be reviewed and may need to be altered according to the child's growth. There is often a problem of accessing healthcare and medication in SA. These problems, although not always easy to solve, must be addressed in patients presenting with disease flares. ${ }^{[17]}$ SLE patients are at an increased risk of infections - these should be actively sought for in any patient presenting with a disease flare.

\section{Disease monitoring}

Deciding whether a child with SLE has a greater or lesser degree of disease activity and determining how to avoid disease damage are essential for patient management. It is not possible to use one individual clinical sign or laboratory value to measure the progression of the disease. To help with this, indices of disease activity and damage have been developed for use in adults with SLE; the same indices are being used in children. ${ }^{[14,22]}$

Disease damage can be evaluated using the Systemic Lupus Erythematosus Disease Activity Index (SLEDAI) and the British Isles Lupus Assessment Group (BILAG) score. ${ }^{[23,24]}$

The only available instrument for measuring disease damage is the SLICC/ACR Damage Index, which looks at both disease-specific

\section{Table 3. Adjuvant therapy}

\begin{tabular}{|c|c|}
\hline Therapy & Explanation \\
\hline Antimalarials, e.g. hydroxychloroquine & $\begin{array}{l}\text { One of the most important recent advances in SLE management has been that all children with the } \\
\text { condition must be treated with hydroxychloroquine. It plays a role in disease modification, helps with } \\
\text { steroid sparing and can reduce the risk of disease flares. It is also associated with higher rates of } \\
\text { renal response and fewer renal relapses } \\
{[8,18,19]}\end{array}$ \\
\hline NSAIDs & $\begin{array}{l}\text { NSAIDs have a role in reducing inflammation and giving symptomatic pain relief, especially for those } \\
\text { with predominant musculoskeletal involvement. Care must be taken due to the adverse side-effect profile, } \\
\text { particularly when dealing with children with } \mathrm{LN} \text {, where these drugs may exacerbate renal disease }{ }^{[18]}\end{array}$ \\
\hline Skin protection & $\begin{array}{l}\text { Skin protection from sunlight with specific clothing and high-factor sunscreen is critical, especially in a } \\
\text { climate as in SA, and will reduce symptoms and long-term skin damage }{ }^{[18,19]}\end{array}$ \\
\hline $\begin{array}{l}\text { Vitamin and mineral supplementation, } \\
\text { especially vitamin D }\end{array}$ & $\begin{array}{l}\text { Essential to the general health of the patient. Vitamin D is most important in patients with } \\
\text { photosensitivity, but should be given to all children with SLE, as it has recently been shown to have } \\
\text { immunomodulatory effects }{ }^{[18,19]}\end{array}$ \\
\hline Aspirin/anticoagulation & $\begin{array}{l}\text { Patients with SLE and positive antiphospholid antibodies but without a previous thrombotic event should } \\
\text { receive low-dose aspirin. Patients with definite antiphospholipid syndrome and a previous thrombotic } \\
\text { event require anticoagulation therapy, targeting an international normalised ratio of } 2.0-3.0^{[19]}\end{array}$ \\
\hline $\begin{array}{l}\text { Angiotensin-converting enzyme } \\
\text { inhibitors }\end{array}$ & Should be used to manage hypertension and proteinuria to reduce the risk of further organ damage ${ }^{[18]}$ \\
\hline Vaccinations & $\begin{array}{l}\text { Routine vaccination schedules should be used to keep children up to date with their immunisations, } \\
\text { although caution is advised with regard to live vaccines in severely immunosuppressed children. Infections } \\
\text { are the most common cause of death in patients with lupus, and pneumococcal infection is particularly } \\
\text { prevalent. Therefore, pneumococcal and annual influenza vaccination should be encouraged }{ }^{[1]}\end{array}$ \\
\hline Tuberculosis screening and prevention & $\begin{array}{l}\text { Tuberculosis is a highly prevalent condition in SA and a high index of suspicion is needed in lupus } \\
\text { patients, especially if they are on immunosuppressive therapy. Regular screening may be necessary, } \\
\text { depending on the disease prevalence and immune status of the patient. Isoniazid prophylaxis may be } \\
\text { required in exposed at-risk patients }\end{array}$ \\
\hline ESRD & $\begin{array}{l}\text { Renal dialysis and renal transplantation are options for patients with ESRD and transplantation has } \\
\text { been shown to be effective in children with } \mathrm{LN} \cdot{ }^{[19]} \text { However, this results in a huge financial strain on an } \\
\text { already over-burdened health system, and with the previous treatment, the aim is to halt the progression } \\
\text { to ESRD in patients with LN }\end{array}$ \\
\hline
\end{tabular}


and non-disease-specific damage and quantifies non-reversible cumulative damage that has occurred since the onset of the disease. ${ }^{[22]}$

\section{Mortality}

In SLE, there is a bimodal pattern of mortality, either from initial disease activity or from complications relating to either the disease itself or therapies used in the treatment of the disease. ${ }^{[25]}$ Renal disease is one of the major poor prognostic factors. ${ }^{[4]}$ Recent improvements in early diagnosis, recognition of milder forms and improved management strategies account for the improvement in mortality in cSLE. In 1981, the 5-year survival rate for cSLE was reported as $82 \%$, but in some more recent studies this figure has increased to $95 \% .{ }^{[13]}$

According to Wadee et al. ${ }^{[26]}$ the 5-year survival probability was between $57 \%$ and $72 \%$ in SA adults. This was thought to be due to an inherently more aggressive disease pattern in black Africans.

\section{When to refer children with lupus}

All SA children with SLE should be referred to a paediatric specialist. If the distance to travel is too far, a telephonic management plan should be made with a paediatric rheumatologist or nephrologist. A paediatric rheumatologist, nephrologist or paediatric specialist with an interest in lupus should see the child as soon as is feasible.

\section{Summary}

- Lupus in children is a rare but serious condition. Recent updates have shown that SLE is being increasingly recognised in southern Africa, particularly in black African children. In childhood SLE, there is a higher proportion of males affected than in adult-onset disease.

- In SA, children present with severe disease, and renal involvement is common.

- The management of cSLE needs to have early specialist input, and there should be access to a MDT.

\section{Key points}

- SLE is becoming increasingly recognised in black SA children.

- In SA, children present with severe disease, particularly with renal disease.

- Every patient with suspected lupus should have their urine analysed for protein and blood.

- A structured approach to investigation and management can help the overall care of the child.

- All children with SLE should be referred to a paediatric rheumatologist, nephrologist or paediatric specialist with knowledge of cSLE.

- A multidisciplinary approach to management is essential.

\section{References}

1. Cassidy JT, Petty RE, Laxer RM, Lindsley L.Textbook of Pediatric Rheumatology. 6th ed. Philadelphia: WB Saunders, 2011:315-318.

2. Danchenko N, Satia JA, Anthony MS. Epidemiology of systemic lupus erythematosus: A comparison of worldwide disease burden. Lupus 2006;15:308-318. [http://dx.doi.org/10.1191/0961203306lu2305xx]

3. Pons-Estel GJ, Alarcón GS, Scofield L, Reinlib L, Cooper GS. Understanding the epidemiology and progression of systemic lupus erythematosus. Semin Arthritis Rheum 2010;39:257-268. [http://dx.doi. progression of systemic lupus erythemato
$\mathrm{org} / 10.1016 /$ j.seminarthrit.2008.10.007]

4. Cervera R, Khamashta MA, Font J, et al., and the European Working Party on Systemic Lupus Erythematosus. Systemic lupus erythematosus: Clinical and immunologic patterns of disease Erythematosus. Systemic lupus erythematosus: Clinical and in
expression in a cohort of 1000 patients. Medicine 1993;72:113-124.

5. Hiraki LT, Benseler SM, Tyrell PN, Harvey E, Hebert D, Silverman E. Ethnic differences in pediatric
. systemic lupus erythematosus. J Rheumatol 2009;36:2539-2546. [http://dx.doi.org/10.3899/jrheum.081141] 6. Khuffash FA, Majeed HA, Lubani MM, Najdi KN, Gunawardana SS, Bushnaq R. Epidemiology of juvenile chronic arthritis and other connective tissue diseases among children in Kuwait. Ann Trop Paediatr 1990;10:255-259.

7. Tiffin N, Hodkinson B, Okpechi I. Lupus in Africa: Can we dispel the myths and face the challenges? Lupus [Epub 30 October 2013] [http://dx.doi.org/10.1177/0961203313509296]

8. Midgley A, Watson L, Beresford MW. New insights into the pathogenesis and management of lupus in children. Arch Dis Child 2014;99:563-567. [http://dx.doi.org/10.1136/archdischild-2013-304397] 9. Hochberg MC. Updating the American College of Rheumatology revised criteria for the classification of systemic lupus erythematosus. Arthritis Rheum 1997;40:1725. [http://dx.doi.org/10.1002/art.1780400928]

10. Petri M, Orbai A-M, Alarcon GS, et al. Derivation and validation of the systemic lupus internation collaborating clinics classification criteria for systemic lupus erythematosus. Arthritis Rheum collaborating clinics classification criteria for systemic
2012;64:2677-2686. [http://dx.doi.org/10.1002/art.34473]

11. Faller G, Thomson PD, Kala UK, Hahn D. Demographics and presenting clinical features of childhood Faller G, Thomson PD, Kala UK, Hahn D. Demographics and
systemic lupus erythematosus. S Afr Med J 2005;95:424-427.

12. Lewandowski L, Schanberg L, Thielman N, Scott C. PULSE - Pediatric Update on Lupus in South Africa: Epidemiology and Management. American College of Rheumatology meeting, November 2014, Boston, MA, USA.
E. 3. Gonzalez B, Hernandez P, Olguin $\mathrm{H}$, et al. Changes in the survival of patients with systemic lupu erythematosus in childhood: 30 years experience in Chile. Lupus 2005;14:918-923. [http://dx.doi. org/10.1191/0961203303lu2183xx]

14. Hiraki LT, Benseler SM, Tyrrell PN, Hebert D, Harvey E, Silverman ED. Clinical and laboratory characteristics and long-term outcome of pediatric systemic lupus erythematosus: A longitudinal study. J Pediatr 2008;152:550-556. [http://dx.doi.org/10.1016/j.jpeds.2007.09.019]

15. Cozzani E, Drosera M, Gasparini G, Parodi A. Serology of lupus erythematosus: Correlation between immunopathological features and clinical aspects. Autoimmune Dis 2014;2014:321359. [http://dx.doi org/10.1155/2014/321359] [Epub 6 February 2014]

16. Yaniv G, Twig G, Shor DB, et al. A volcanic explosion of autoantibodies in systemic lupus erythematosus: A diversity of 180 different antibodies found in SLE patients. Autoimmun Rev 2015;14: erythematosus: A diversity of 180 different antibodies for

17. Lewandowski LB, Scott C. Apartheid and healthcare access for paediatric systemic lupus erythematosus patients in South Africa. S Afr J Child Health 2015;9:36-37. [http://dx.doi.org/10.7196/sajch.869]
. 18. Bertsias G, Ioannidis JPA, Boletis J, et al. EULAR recommendations for the management of systemic lupus
patients in South Africa. A Ar J Child Health 2015;936-37. http://x.doi.org/10.7196/sach.869] B. Bertsias G, Ioannidis JPA, Boletis J, et al. EULAR recommendations for the management of systemic lupus
erythematosus. Report of a Task Force of the EULAR Standing Committee for International Clinical Studies Including Therapeutics. Ann Rheum Dis 2008;67: 195-205. [http://dx.doi.org/10.1136/ard.2007.070367]

19. Arici ZS, Batu ED, Ozen S. Reviewing the recommendations for lupus in children. Curr Rheumatol Rep 2015;17(3):17. [http://dx.doi.org/10.1007/s11926-014-0489-5]

20. Tarr G, Hodkinson B, Reuter H. Superheroes in autoimmune warfare: Biologic therapies in current South African practice. S Afr Med J 2014;104:787-791. [http://dx.doi.org/10.7196/SAMJ.8947]

21. Heijstek MW, de Bruin LMO, Bijl M, et al. EULAR recommendations for vaccination in paediatric patient with rheumatic diseases. Ann Rheum Dis 2011;70:1704-1712. [http://dx.doi.org/10.1136/ard.2011.150193]

22. Gladman DD, Ginzler E, Goldsmith C, et al. The development and initial validation of the Systemic Lupus International Collaborating Clinics/American College of Rheumatology Damage Index for systemic International Collaborating Clinics/American College of Rheumatology Damage Index for system

23. Bombardier C, Gladman DD, Urowitz MB, Caron D, Chang CH. Derivation of the SLEDAI: A disease activity 3. Bombardier C, Gladman DD, Urowitz MB, Caron D, Chang CH. Derivation of the SLEDAI: A disease acti
index for lupus patients. Arthritis Rheum 1992;35:630-640. [http://dx.doi.org/10.1002/art.1780350606]

index for lupus patients. Arthritis Rheum 1992;35:630-640. [http://dx.doi.org/10.1002/art.1780350606]
24. Brunner HI, Feldman BM, Bombardier C, Silverman ED. Sensitivity of the Systemic Lupus

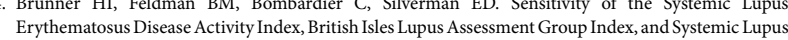
Erythematosus Disease Activity Index, British Isles Lupus Assessment Group Index, and Systemic Lupus
Activity Measure in the evaluation of clinical change in childhood onset systemic lupus erythematosus. Activity Measure in the evaluation of clinical change in childhood onset systemic lupus ery
Arthritis Rheum 1999;42:1354-1360. [http://dx.doi.org/10.1002/1529-0131(199907)42:7]

25. Urowitz MB, Bookman AA, Koehler BE, Gordon D, Smythe HA, Ogryzlo MA. The bimodal pattern of systemic lupus erythematosus. Am J Med 1976;60:221-225. [http://dx.doi.org/10.1016/0002-9343(76)90431-9]

26. Wadee S, Tikly M, Hopley M. Causes and predictors of death in South Africans with systemic lupus erythematosus. Rheumatology 2007;46:1487-1491. [http://dx.doi.org/10.1093/rheumatology/kem180]

\section{Continuing medical education resources}

ACR criteria. http://www.rheumatology.org/Portals/0/Files/1997\%20Update\%20of\%201982\%20Revised. pdf (accessed 13 November 2015).

SLICC criteria. http://www.rheumtutor.com/2012-slicc-sle-criteria/ (accessed 13 November 2015). SLICC/ACR Damage Index. http://www.ncbi.nlm.nih.gov/pubmed/10685799 (accessed 13 November 2015). 